\title{
Téoros
}

Revue de recherche en tourisme

\section{Les Allemands au Québec}

Un marché de niches?

\section{Jean Stafford et Bruno Sarrasin}

Volume 17, numéro 2, été 1998

URI : https://id.erudit.org/iderudit/1072282ar

DOI : https://doi.org/10.7202/1072282ar

Aller au sommaire du numéro

Éditeur(s)

Université du Québec à Montréal

ISSN

0712-8657 (imprimé)

1923-2705 (numérique)

Découvrir la revue

\section{Citer cette note}

Stafford, J. \& Sarrasin, B. (1998). Les Allemands au Québec : un marché de niches ? Téoros, 17(2), 53-55. https://doi.org/10.7202/1072282ar d'utilisation que vous pouvez consulter en ligne.

https://apropos.erudit.org/fr/usagers/politique-dutilisation/ 


\section{$+\ldots$ \\ Les Allemands au Québec \\ UN MARCHÉ DE NICHES?}

\section{Jean Stafford et}

\section{Bruno Sarrasin}

Cette chronique porte sur la conjoncture touristique. Elle propose un portrait simplifie de la situation du tourisme à partir d'une clientéle précise et des données disponibles. Les notions usuelles de l'analyse prévisionnelle seront utilisées : la tendance (passée, présente et future), la saisonnalité, le cycle et les mouvements irréguliers. Les prévisions seront faites pour les cinq prochaines années.

L'Allemagne représente le plus important pays européen émetteur de touristes. Il suffit de voyager en Europe, en Asie, en Océanie et dans les Amériques pour le constater. Bien au-dessus de la moyenne des pays industrialisés, $78 \%$ de la population allemande quittait son domicile pour des vacances de cinq jours et plus en 1995 (Lettl-Schröder, 1998). Au Québec, la moitié de la population prenait des vacances en 1995, ce qui représente un taux de départ beaucoup plus faible que celui des Allemands ${ }^{1}$. La conjoncture économique des années 1990 n'a cependant pas épargné ce pays qui doit, en plus d'assumer les coûts de sa réunification et la réduction de sa dette, réaliser les critères de convergence nécessaires à la création d'une monnaie européenne unique.

Ces conditions affectent bien entendu le marché du travail, les salaires et la consommation. La demande touristique étant très sensible à la conjoncture économique (réelle ou anticipée), il n'est pas surprenant de constater une baisse de $8 \%$ du départ en vacances des Allemands en 1996 par rapport à l'année précédente. La chute du taux de départ s'est maintenue depuis, avec $3 \%$ de moins en 1997 qu'en 1996. Cette perte de 2 millions de voyageurs au cours des deux dernières années concerne principalement les Allemands voyageant chez eux (particulièrement en Allemagne de l'Est) et les voyages individuels, affectant peu les voyages vers l'étranger. Plus de 42,8 millions d'Allemands prenaient des vacances en 1997 (Lettl-Schröder, 1998).

Le Québec semble représenter peu de choses pour ce marché, qui comptait pour seulement $8,7 \%$ des touristes européens et $6,7 \%$ des touristes étrangers (sauf les États-Unis) en 1997. L'Allemagne représente le troisième marché d'Europe pour le Québec, après la France et le RoyaumeUni. En comparaison avec ces deux pays, les Allemands séjournent moins longtemps et ont une dépense par séjour parmi les moins élevés. Ils voyagent surtout sans enfants ( $77,1 \%$ d'entre eux en 1994) et semblent considérer le Québec comme l'étape d'un circuit nord-américain qui commence souvent par les États-Unis ou l'Ontario. Cette réalité s'illustre notamment par $100 \%$ de plus de visites-province en Ontario qu'au Québec en 1996 (209 200 contre $107(100)^{2}$. Pour cette même année, 1,7 million d'Allemands voyageaient en Amérique du nord, montrant ainsi que la part relative du Québec peut être non négligeable sur ce marché?

\section{TENDANCES}

L'évolution des arrivées de touristes allemands au Québec est marquée par une croissance moyenne de $4,4 \%$ sur une période de 20 ans, ce qui est relativement peu en comparaison à d'autres marchés européens comme celui des Français (tableau 1). Certes, le nombre d'Allemands entrant au Canada via le Québec a plus que doublé depuis 1978, mais à l'exception de l'année record de 1996, les arrivées ont été ponctuées de plusieurs reculs jusqu'au milieu des années 1990. Ces conditions auraient sans doute été différentes si la gestion canadienne des ressources aéroportuaires n'avait poussé Lufthansa à cesser ses vols directs sur Montréal au profit de Toronto.

Contrairement à d'autres clientèles touristiques davantage affectées par la conjoncture économique, les Britanniques par exemple ${ }^{4}$, les Allemands affichent une croissance presque linéaire de 1985 à 1994 (voir graphique 1). Malgré une baisse marquée en 1983, 1985 et 1997, les Allemands qui choisissent le Québec comme porte d'entrée canadienne sont en constante augmentation. La plupart d'entre eux, comme les Nord-Américains, cherchent d'abord le soleil, la plage et un environnement de relaxation ${ }^{5}$. Puisque le Québec n'a pas de plage à offrir (au sens du Beach holiday), il doit se positionner sur des caractéristiques fortes (culture, nature, aventure), valorisant ainsi le caractère lointain (long haul) de la destination.

Ce sont surtout de jeunes couples d'Allemands ${ }^{6}$ qui viennent au Québec par agrément ${ }^{7}$. Cette clientèle, pour qui la valeur élevée du mark par rapport au dollar 
américain a longtemps favorisé le départ vers l'étranger, pourrait se diviser en deux grandes catégories : les voyageurs à petit budget, qui contribuent a la baisse du niveau moyen des dépenses par séjour et les voyageurs de « niches qui, comme certains articles de ce numéro l'ont évoqué, recherchent une expérience unique et sont disposés à investir en conséquences.

C'est bien entendu cette dernière clientèle qui devrait intéresser les intervenants touristiques du Québec, non seulement parce qu'elle est lucrative, mais parce qu'elle contribue à positionner le Québec comme destination * unique $*$, avec le tourisme autochtone par exemple, ajoutant ainsi un avantage concurrentiel important à la destination. En comparaison à nos marches primaires du nord-est américain, les Allemands prendront difficilement le titre de * touristes de masse $*$ où la rentabilité passe par le volume. Autant miser sur les particularités de leur demande et présenter un produit distinctif de qualité.

\section{SAISONNALITÉ}

Comme l'ensemble des clientèles touristiques du Québec, les arrivées d'Allemands sont fortement marquées par la saisonnalité (tableau 2). Sur une période de 20 ans (1978-1997) on constate une concentration des arrivées aux mois de juin, juillet, aout et septembre, particulièrement depuis les cinq dernières années. Depuis 1978 , on peut observer que la fréquentation des mois de janvier à mars s"est réduite au profit des mois de septembre et octobre dont la croissance représente respectivement $73 \%$ et $46 \%$ de 1978 1982 à 1993-1997.

Ces conditions reflètent un déplacement généralisé de la demande touristique européenne des mois de juin, juillet et août, au profit des deux mois suivants. Les touristes européens en auraient-ils assez de la haute saison ou seraient-ils tout simplement en train de la déplacer? Même si la période d'été représente toujours l'essentiel des arrivées d'Allemands au Québec, la fréquentation de 1978-1982 à $1993-1997$ a diminué de $15,4 \%$ en juin, $19 \%$ en juillet et $4,7 \%$ en août. Le trimestre d'hiver semble encore plus délaissé qu'auparavant, faisant des Allemands une clientêle de plus en plus concentrée sur l'été-automne.

\begin{tabular}{|c|c|}
\hline \multicolumn{2}{|c|}{ TABLEAU 1 } \\
$\begin{array}{c}\text { Les arrivées des touristes d'Allemagne au Québec ; les taux d'accroissement annuels } \\
\text { moyens (moyenne géométrique), de } 1978 \text { à } 1997 \text { par période dé cinq années }\end{array}$ \\
\hline Années & $\begin{array}{c}\text { Taux d'accroissement } \\
\text { en \% }\end{array}$ \\
\hline $1978-1982$ & 4,9 \\
\hline $1983-1987$ & 5,8 \\
\hline $1988-1992$ & 3,4 \\
\hline $1993-1997$ & 4,5 \\
\hline $1978-1997$ & 4,4 \\
\hline
\end{tabular}

Source: nos calculs a partir des donnetes de Statistique Canada.

\section{TABLEAU 2}

Les arrivées des touristes d'Allemagne au Québec; l'évolution des coefficients saisonniers" de 1978 à 1997 par période de cinq années

\begin{tabular}{|l|c|c|c|c|c|c|c|c|c|c|c|c|}
\hline \multirow{2}{*}{ ANNÉES } & \multicolumn{10}{|c|}{ MOIS } \\
\cline { 2 - 13 } & $\mathbf{1}$ & $\mathbf{2}$ & $\mathbf{3}$ & $\mathbf{4}$ & $\mathbf{5}$ & $\mathbf{6}$ & $\mathbf{7}$ & $\mathbf{8}$ & $\mathbf{9}$ & $\mathbf{1 0}$ & $\mathbf{1 1}$ & $\mathbf{1 2}$ \\
\hline $1978-1982$ & 35 & 39 & 52 & 56 & 106 & 157 & 237 & 199 & 126 & 94 & 44 & 52 \\
\hline $1983-1987$ & 34 & 44 & 51 & 65 & 114 & 143 & 216 & 202 & 134 & 90 & 47 & 59 \\
\hline $1988-1992$ & 35 & 36 & 52 & 62 & 105 & 137 & 219 & 182 & 171 & 101 & 43 & 56 \\
\hline $1993-1997$ & 27 & 27 & 41 & 48 & 108 & 136 & 199 & 190 & 218 & 137 & 29 & 40 \\
\hline Moyennes & 33 & 36 & 49 & 58 & 108 & 143 & 218 & 193 & 162 & 105 & 41 & 52 \\
\hline
\end{tabular}

1. Ces coefficienss sont calculés à partir de la méthode de adécomposition des series chronologiques: Al l'aide du logiciel SPSS, ea utilisant les donmés de Statistique Canada.

\section{TABLEAU 3}

Les arrivées des touristes d'Allemagne au Québec, de 1978 à 1997 ; synthèse des éléments de décomposition de la série chronologique

\begin{tabular}{|c|c|}
\hline Tendance: & $\begin{array}{l}\text { Tendance a moyenne * (autour de } 4 \% \text { ) mais assez constante } \\
\text { (sauf pour la période 1988-1992). La croissance est soutenue, } \\
\text { c'est donc un marché intéressant à moyen terme et à long terme } \\
\text { même si le volume réel de touristes est faible. }\end{array}$ \\
\hline Saisonnalité : & $\begin{array}{l}\text { Il y a de très fortes variations de la saisonnalité (comme toutes } \\
\text { les clientèles européennes) ; les coefficients saisonniers } \\
\text { évoluent de } 27 \text { à } 237 \text { selon les mois et les années*; les mois } \\
\text { les plus a forts o sont : juin, juillet, août et septembre. }\end{array}$ \\
\hline Cycle: & $\begin{array}{l}\text { Un cycle très accentué qui oscille de } 59 \text { à } 162 \\
\text { (sur une base de 100). }\end{array}$ \\
\hline Mouvements irréguliers : & $\begin{array}{l}\text { Les mouvements aléatoires restent dans une fourchette } \\
\text { tolérable }( \pm 9 \%)\end{array}$ \\
\hline
\end{tabular}

* Vair le tableau 2. 


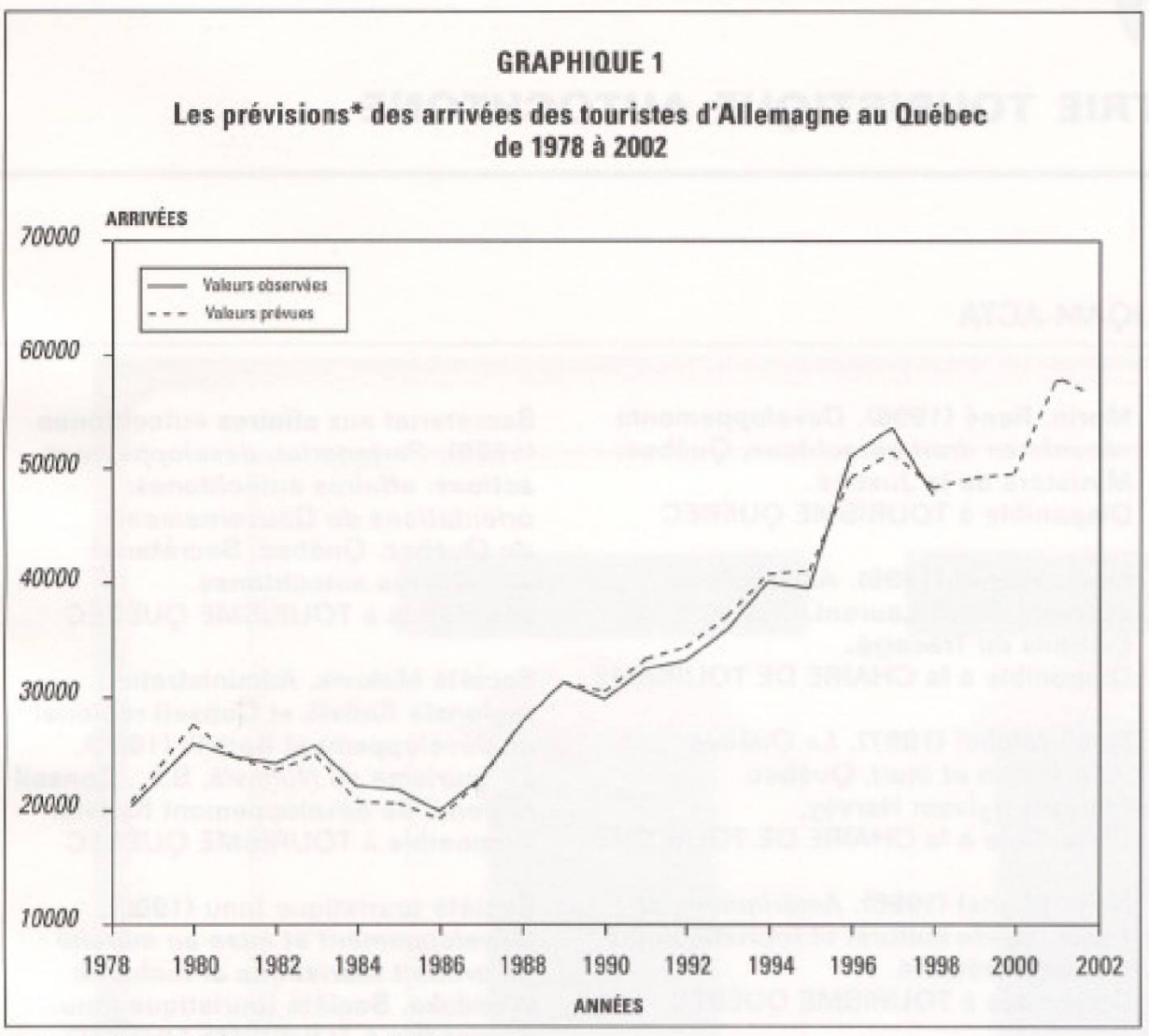

* Une tendance linéaire avec un cycle conjoncturel.

\section{LES ALLEMANDS : UNE CLIENTĖLE POUR LE QUÉBEC?}

Le marché allemand est et demeurera en croissance à moyen terme pour le Québec: Beaucoup plus a stables $»$ que celles des Britanniques, les arrivées de touristes allemands pourraient atteindre le seuil des 60000 à l'horizon de 2002 et ce, malgré un cycle négatif pour 1998 et 1999 (graphique 1). Malgré des prévisions économiques peu reluisantes à moyen terme, il semble que les Allemands, en particulier pour les groupes sociaux les plus stables financièrement ${ }^{10}$, continueront de voyager à l'étranger avec encore plus d'intérêt. En 1997, une enquête de GfK a montré qu'en 1998, les ménages allemands étaient prêtts à augmenter leur budget de voyage de $5 \%$ par rapport à 1996, 27 \% d'entre eux prévoyant dépenser plus de 5000 marks pour leur vacances (Lettl-Schröder 1998 : 44).

Malgré une santé financière relativement bonne d'ici l'an 2000, marquée par une stabilité des prix et le maintien du surplus de la balance commerciale, les conditions économiques des ménages demeureront, au mieux, inchangées pour les deux pro-

\section{NOTES}

1 Voir Marcel Samson et Jean Stafford (1996), Vacances et tourisme en 1995. Enquète auprès d'un échantillon de Québécois et de Montréalais sur le comportement des vacances, Québec, ministère des Affaires municipales.

2 Les données sont de Statistique Canada.

3 La comparaison des données (1,7 million et 107 100) doit être faite sous réserve de leur source différente (Lettl-Schröder, 1998 et Statistique Canada).

4 Voir à ce sujet Stafford, Jean et Bruno Sarrasin (1998), «Les Britanniques au Québec. Quel tourisme? s, Téoros, vol. 17, $\mathrm{n}^{\circ} \mathrm{I}$, p. 61-63.

5 Selon Lettl-Schröder (1998: 38 ), $56 \%$ des Allemands voyageant à l'étranger en 1996 cherchaient le soleil et la plage (Beach holiday).

6 En 1994, 31 \% des touristes (visites-province) en provenance de l'Allemagne avaient de 24 a 34 ans et $41,8 \%$ des groupes de voyageurs étaient composés de deux adultes (Tourisme Québec, 1995: 42-43).

7 En 1994, 55,2 \% des touristes allemands visitaient le Québec par agrément (Tourisme Québec, 1995: 42-43).

8 Selon Lettl-Schroder (1998 : 38-39), en 1996 $12 \%$ des voyageurs allemands vers l'étranger $s$ 'intéressaient au séjour d'aventure.

9 Voir tableau 3 et graphique 1.

10 Les jeunes-vieux de David Foot, babyboomers et jeunes retraités représentent un marché lucratif en quête d'originalité et sans contraintes de temps. etre segmenté. Pour éviter une offre indifférenciée présentant le Québec comme une destination où le fait français suffirait à motiver non seulement un séjour, mais 1'arrivée en Amérique via le Québec, les intervenants touristiques devraient miser sur l'originalité et la qualité. Les initiatives de valorisation du tourisme autochtone, dont ce numéro fait état, ne constituent pas l'unique voie de développement mais représentent un pas de plus dans le positionnement unique du Québec comme destination internationale.

Jean Stafford est professeur au Département d'études urbaines et touristiques de l'UQAM.

Bruno Sarrasin est chargé de cours au Département d'études urbaines et touristiques de I'UQAM.

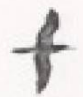

\section{BIBLIOGRAPHIE}

Commission canadienne du tourisme (1997), Programme de marketing de l'Europe. Plan opérationnel $1997 / 98$, juillet.

Commission canadienne du tourisme (1998), site Internet http:/206.191.33.50, consulté le 8 juin 1998.

Lettl-Schröder, Maria (1998), * Germany Outbound $*$,Travel \& Tourism Analyst, $\mathrm{n}^{\circ} 1$, p. 2645.

Tourisme Québec (1995), Les touristes des pays autres que les Etats-Unis au Quebec, Statistiques touristiques, Québec, novembre.

Tourisme Québec (1997), Le tourisme au Québec en 1995. Une réalité économique importante, Statistiques touristiqués, Québec, mars. 